\title{
EDITORIAL
}

\section{Natriuretic peptide measurements as part of the diagnostic work-up in pleural effusions: an emerging concept?}

\author{
T. Mueller* and M. Haltmayer*,\#
}

I $\mathrm{n}$ the 1950s and 60s, the possible role of the heart in the control of renal excretion of salt and water, and thereby of the extra cellular fluid volume, as well as the presence of specific granules within atrial muscle cells was recognised [1, 2]. Nevertheless, little attention was paid to the possibility of the existence of an endocrine cardiac natriuretic hormone system until the landmark experiments in the late 1970s by DE BoLD et al. [3], which led to the discovery of atrial natriuretic peptide (A-type natriuretic peptide; ANP) having potent natriuretic, diuretic and vasodilatator actions.

This initiated intense research characterising a family of structurally similar but genetically distinct natriuretic peptides, including B-type natriuretic peptide (BNP, formerly named brain natriuretic peptide), C-type natriuretic peptide $(\mathrm{CNP})$, dendroaspis natriuretic peptide and urodilatin. ANP and BNP are synthesised by cardiac myocytes (so-called cardiac natriuretic peptides). It is now generally accepted that their release into the blood is increased by factors that raise intra-cardiac pressure and volume overload [4]. Unlike ANP, the primary source of BNP is considered to be the cardiac ventricles. Other natriuretic peptides, such as CNP and urodilatin, are not secreted by cardiomyocytes but by other tissues.

Cardiac natriuretic peptides are derived from pre-prohormones [4]. In particular, BNP is derived from the precursor pre-proBNP, which contains 134 amino acids including a signal peptide of 26 amino acids. The prohormone containing 108 amino acids (proBNP), produced by cleavage of the signal peptide, is further split into BNP, which is considered to be the biologically active hormone, and an inactive amino terminal fragment (NT-proBNP). BNP and NT-proBNP are secreted on an equimolar basis, but their molar ratio in plasma is not 1:1 because BNP has a shorter plasma half-life than NT-proBNP.

As indicated, the natriuretic peptide hormones regulate blood pressure, electrolyte balance and fluid volume. These actions

*Dept of Laboratory Medicine, Konventhospital Barmherzige Brueder, Linz, and ${ }^{\#}$ Paracelsus Private Medical University, Salzburg, Austria.

CORRESPONDENCE: T. Mueller, Dept of Laboratory Medicine, Konventhospital Barmherzige Brueder, Seilerstaette 2-4, A-4020 Linz, Austria. Fax: 43 73276773799. E-mail: thomas.mueller@ bs-lab.at are counterbalanced by the vasoconstrictive and sodiumretaining actions of the renin-angiotensin-aldosterone system. These two regulatory systems help to maintain sodium and fluid volume homeostasis in a healthy cardio-renal environment [4]. If atrial and ventricular pressure reaches a state of pathological stretch, ANP, BNP and the amino terminal fragments of their precursor hormones (i.e. NT-proANP and NT-proBNP, respectively) are released into the blood. As the severity of heart failure increases, so do cardiac stretch and the amount of the peptides in a patient's blood. Therefore, in principle, the measurement of ANP/NT-proANP and BNP/ NT-proBNP concentrations may aid decision making in a variety of clinical settings (e.g. diagnosis of heart failure in symptomatic patients, monitoring of treatment in heart failure, prognostic marker in heart failure patients, etc.) [5]. However, in general, BNP and NT-proBNP are believed to have a better diagnostic and prognostic potential than ANP and NTproANP. Hence, during the last few years, most manufacturers have focused on the development of point-of-care and fully automated assays measuring circulating BNP and NT-proBNP concentrations for use in clinical practice.

In the present issue of the European Respiratory Journal, the paper by KolDITZ et al. [6] addresses the well-known challenge of diagnosing heart failure as the cause of a pleural effusion. The authors evaluated the diagnostic accuracy of NT-proBNP measured in serum and pleural fluid (index tests) for the identification of pleural effusions caused by heart failure in 93 patients referred for thoracentesis. The reference standard was the final clinical diagnosis established by reviewing the hospital records of all patients. An important observation was that serum and pleural fluid NT-proBNP concentrations correlated closely. Both were significantly higher in patients with effusions caused by heart failure than in patients with pleural effusions attributable to other causes. NT-proBNP cutoff concentrations of $4,000 \mathrm{ng} \cdot \mathrm{L}^{-1}$ for both serum and pleural fluid had sensitivities and specificities of $\sim 90$ and $93 \%$, respectively, for the diagnosis of heart failure. By performing receiver operating characteristic (ROC) curve analyses for NTproBNP in both specimen types, KolDITzet al. [6] found similar areas under the curve of 0.98 for the diagnosis of heart failure in their study sample. Accordingly, they concluded that measurement of NT-proBNP in patients with pleural effusions of unknown origin may be of assistance in the diagnosis of heart failure as the underlying cause. 
The diagnosis of pleural effusions caused by heart failure is usually made clinically, supported by finding a transudate when pleural fluid is examined. Due to their high sensitivity in identifying exudates, the criteria proposed in 1972 by LiGHT et al. [7] have become the standard method for making a distinction between transudates and exudates. Typical diseases with transudative pleural effusions are heart failure, cirrhosis, nephrosis and hypoalbuminaemia, whereas diseases such as malignancy, tuberculosis, infection and pancreatitis are considered to cause exudative pleural effusions [8, 9]. Light's criteria dichotomise effusions into exudative or transudative categories using three pleural test criteria: 1) pleural fluid/ serum protein ratio (cut-off 0.5); 2) pleural fluid lactate dehydrogenase (LDH) concentration (cut-off $200 \mathrm{IU} \cdot \mathrm{L}^{-1}$ or $>2 / 3$ upper limit of normal for serum); and 3) pleural fluid/ serum LDH ratio (cut-off 0.6) [8,9]. If one of these cut-off values is exceeded, the effusion is considered to be an exudate. The main disadvantage of this classification, however, appears to be the occurrence of an exudative range of protein levels in a distinct rate of patients with heart failure, especially in the setting of diuretic use. This may lead to unnecessary investigations being carried out in these patients. As a consequence, several other pleural fluid test criteria, including pleural fluid protein concentration, pleural fluid cholesterol concentration and pleural fluid/serum cholesterol ratio, have been investigated, but none of these test criteria proved to be advantageous compared with Light's criteria [9]. Therefore, additional diagnostic tests, such as BNP or NT-proBNP measurements, may represent an advance in the diagnostic work-up of pleural effusions. Indeed, recently some rather preliminary studies have been published supporting this proposed approach [10-12].

Despite the impressive test characteristics of serum and pleural fluid NT-proBNP in the diagnosis of heart failure as the cause of a pleural effusion, as described in the study by KoLDITz et al. [6], there are some study design features and methodological points that warrant cautious interpretation of the results, as recognised by the authors. First, they indicate that both patients referred for diagnostic and therapeutic thoracenteses were enrolled into the study. As this is a study investigating the diagnostic accuracy of NT-proBNP for evaluation of pleural effusions of unknown origin, it is probably biased in terms of disease prevalence and, thus, cut-off values and the diagnostic accuracy of NT-proBNP measurements. Secondly, although the reference standard was assessed blinded to the biochemical data, the attending physicians were not blinded to the NT-proBNP results. Therefore, it is conceivable that they used this information for further diagnostic approach and the treatment strategy of their patients. Thirdly, the study design, excluding patients in whom a definite diagnosis could not be established, is widely used for studies investigating the cause of pleural effusions. However, the exclusion of these patients may have produced ROC curves overestimating the real diagnostic utility of serum and pleural fluid NT-proBNP in this setting. Finally, no conclusions can be derived from the study as to what extent NT-proBNP measurements in serum or pleural fluid add diagnostic information to clinical variables (e.g. age, history of heart failure or myocardial infarction, unilateral effusion, and left ventricular ejection fraction) or to the treating clinician's initial diagnostic impression.
Nevertheless, the study by KOLDITZ et al. [6] demonstrated a strong correlation of NT-proBNP concentrations in serum and pleural fluid and equal diagnostic accuracies for serum and pleural fluid NT-proBNP measurements for the discrimination of pleural effusions attributable to heart failure. Therefore, it could be claimed that it may not be necessary to perform diagnostic thoracenteses in patients with serum NT-proBNP concentrations exceeding a cut-off value with a high positive predictive value, making the diagnosis of heart failure as the underlying cause of the pleural effusion very likely in these patients. However, in all other patients with serum NTproBNP concentrations below such a cut-off value, early diagnostic thoracentesis may be necessary to determine whether the effusion is exudative or transudative in nature. However, this proposed diagnostic approach is currently speculative and has to be confirmed by further studies. These studies should evaluate patients with pleural effusions randomised to those receiving and those not receiving serum NT-proBNP determinations, and to compare the perceived need for thoracentesis in the two groups.

In conclusion, the article by KOLDITZ et al. [6] suggests an expanded diagnostic utility for inactive amino terminal fragment-pro B-type natriuretic peptide measurements. The results support the feasibility of using inactive amino terminal fragment-pro B-type natriuretic peptide measurements as part of the diagnostic approach in patients with pleural effusion of uncertain aetiology. However, as outlined previously, additional research is necessary to answer the question of whether natriuretic peptide measurements may emerge as clinically useful in the diagnostic work-up of patients with pleural effusions. During the next few years, we anticipate that appropriately designed studies on this topic will clarify this possibility.

\section{REFERENCES}

1 Henry JP, Gauer OH, Reeves JL. Evidence of the atrial location of receptors influencing urine flow. Circ Res 1956; 4: 85-90.

2 Jamieson JD, Palade GE. Specific granules in atrial muscle cells. J Cell Biol 1964; 23: 151-172.

3 de Bold AJ, Borenstein HB, Veress AT, Sonnenberg H. A rapid and potent natriuretic response to intravenous injection of atrial myocardial extract in rats. Life Sci 1981;28: 89-94.

4 Mair J, Friedl W, Thomas S, Puschendorf B. Natriuretic peptides in assessment of left-ventricular dysfunction. Scand J Clin Lab Invest Suppl 1999; 230: 132-142.

5 Clerico A, Emdin M. Diagnostic accuracy and prognostic relevance of the measurement of cardiac natriuretic peptides: a review. Clin Chem 2004; 50: 33-50.

6 Kolditz M, Halank M, Schiemanck CS, Schmeisser A, Höffken G. High diagnostic accuracy of NT-proBNP for cardiac origin of pleural effusions. Eur Respir J 2006; 28: 144-150.

7 Light RW, Macgregor MI, Luchsinger PC, Ball WC Jr. Pleural effusions: the diagnostic separation of transudates and exudates. Ann Intern Med 1972; 77: 507-513.

8 Light RW. Clinical practice. Pleural effusion. N Engl J Med 2002; 346: 1971-1977.

9 Burgess LJ. Biochemical analysis of pleural, peritoneal and pericardial effusions. Clin Chim Acta 2004; 343: 61-84. 
10 Porcel JM, Vives M, Cao G, Esquerda A, Rubio M, Rivas MC. Measurement of pro-brain natriuretic peptide in pleural fluid for the diagnosis of pleural effusions due to heart failure. Am J Med 2004; 116: 417-420.

11 Tomcsanyi J, Nagy E, Somloi M, et al. NT-brain natriuretic peptide levels in pleural fluid distinguish between pleural transudates and exudates. Eur J Heart Fail 2004; 6: 753-756.

12 Gegenhuber A, Mueller T, Dieplinger B, Lenz K, Poelz W, Haltmayer M. Plasma B-type natriuretic peptide in patients with pleural effusions: preliminary observations. Chest 2005; 128: 1003-1009. 